\title{
Application of Mössbauer spectroscopy for the diagnostics of functional groups of the humic substances
}

Pankratov D.A., Zimbovskaya M.M.

Lomonosov Moscow State University, Moscow, Russia, pankratov@radio.chem.msu.ru

Keywords: coordination binding, probe iron atom, Mössbauer spectroscopy

doi: 10.36291/HIT.2019.pankratov.043

The waters of open reservoirs, soils and organogenic rocks contain high-molecular organic compounds of natural origin - humic substances (HS). HS, having a large number of diverse functional groups, is capable of entering into redox, sorption, and complexing interactions with iron, as one of the most common elements in the Earth's crust.

Earlier, we showed that, HS of different origins and fractional compositions are adsorbed selectively on macroporous weakly basic anion-exchange resin (MWBAER) [1]. So, HS of coal origin are sorbed on MWBAER by predominantly carboxyl groups of aromatic fragments, while lignosulfonate derivatives, by means of strongly acidic sulfonic groups. Thus, in the process of HS sorption on MWBAER, fractionation of individual functional groups of natural polyelectrolytes is carried out, which allows them to be excluded from further interactions, for example, with transition metal ions. It is known that MWBAER by immobilization of HS can be used for sorption of iron ions for natural water treatment. Therefore, it is obvious that 57-iron, being a Mössbauer nuclide, can be used as a probe for structural fragments of HS capable of forming coordination bonds with iron atom.

To demonstrate the possibilities of this approach for studying the fractionation of HS functional groups using MWBAER, MWBAER immobilized by HS after adsorption of 57iron chloride solutions was studied by Mössbauer spectroscopy. Two HS preparations was immobilized in MWBAER: Sakhalinskii potassium humate (OOO Biomir-2000, Moscow, Russia) of coal origin and sodium lignohumate (OOO NPO RET, St. Petersburg, Russia), which is produced via the oxidation of lignin sulfonate. The conditions for sorption of 57iron ions were determined based on previously obtained isotherms of adsorption of iron salts on these sorbents. Mössbauer spectra were obtained both for wet adsorption products frozen to the temperature of liquid nitrogen, and for dried samples at room temperature. The Mössbauer spectra are broadened, asymmetric doublets (Fig. 1), which can be satisfactorily described by a superposition of two or three subspectra of symmetric doublets corresponding to different sites of probe atoms.

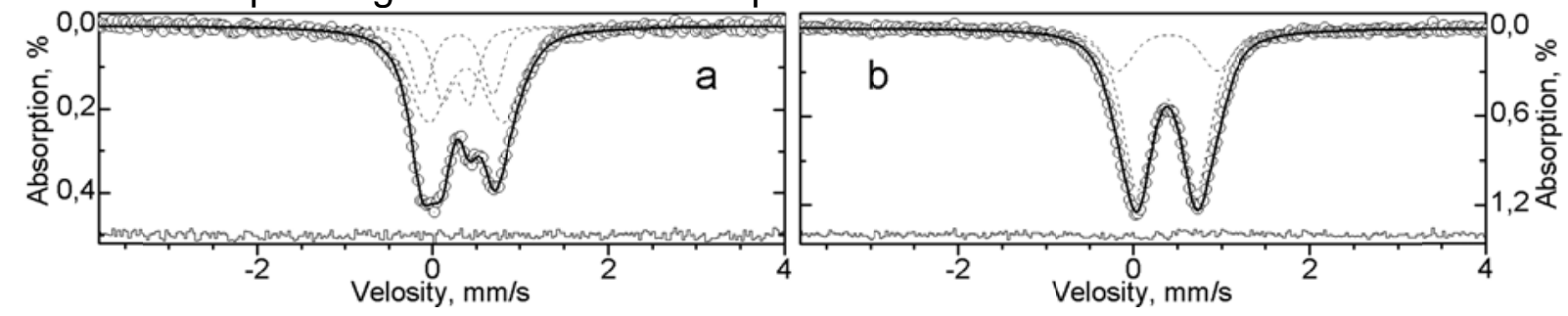

Figure 1. Mössbauer spectra at $298 \mathrm{~K}$ of 57 -iron sorbed on MWBAER immobilized with: a) potassium humate, b) sodium lignohumate.

Thus, the method of fractionation of the functional groups of HS using MWBAER was tested, which allows us to study the nature of the coordination "HS - iron" interaction. It is shown that coordinated HS iron can occupy two or three different positions at the formation of bonds with fractionated by functional groups HS.

References

1. Pankratov D.A. et al. // Russ. J. Phys. Chem. A. 2017. 91(6):1109-1115. 